\title{
Emphysematous Pyelonephritis Treated With Percutaneous Nephrolithotomy
}

\author{
Anthea Ki Kwan Lamª, biacomo Caddeo ${ }^{\mathrm{a}}$
}

\begin{abstract}
Emphysematous pyelonephritis (EPN) has a high rate of mortality due to sepsis as a complication of EPN, and majority of patients have a good outcome with a minimally invasive approach such as medical management by antibiotics and supportive care. Studies show that early nephrectomy should be reserved for those who do not respond to initial conservative management once the patient is stabilized. Decisions of management should take into account the patient's current renal function which can be determined by looking at serum creatinine level or eGFR as to whether nephrectomy, percutaneous drainage or antibiotics would be most appropriate bearing in mind that a minimal approach of antibiotics alone has a higher risk of mortality compared with additional percutaneous drainage of abscess or nephrectomy. Although regarding this case, the patient had a background of congenital renal disease namely her medullary sponge kidney in addition to the formation of matrix stone which increased the severity of her already class IV EPN, conventional treatment via antibiotics was unsuccessful and so was nephrostomy drainage. Therefore unconventional method of percutaneous nephrolithotomy (PCNL) was adopted to treat her bilateral disease and this was successful as evident on the scans.
\end{abstract}

Keywords: Emphysematous pyelonephritis; Percutaneous nephrolithotomy; Nephrectomy

\section{Introduction}

Emphysematous pyelonephritis (EPN) is an acute severe necrotizing infection of renal parenchyma and surrounding tissues that leads to gas in the parenchyma, collecting system or perinephric tissue, a life-threatening condition with a mortality rate of $13.5 \%$ [1]. Incidence is highest in those between 40 and 50 years of age with the female/male ratio of $6: 1[2,3]$.

Manuscript accepted for publication January 17, 2014

aUrology, Royal Derby Hospital, Derby, Derbyshire, DE22 3NE, UK ${ }^{b}$ Corresponding Author: Anthea Ki Kwan Lam, Urology, Royal Derby Hospital, Derby, Derbyshire, DE22 3NE, UK. Email: anthea.lam@nhs.net

doi: http://dx.doi.org/10.14740/wjnu145w
There is speculation that this is due to an association between EPN and urinary tract obstruction which accounts for $25-40 \%$ of cases [1]. The most common cause is the Gram-negative organism, Escherichia coli (70-80\%) which produces anerobic metabolites lactate and glucose leading to carbon dioxide and hydrogen accumulation within the parenchyma $[1,4]$.

Risk factors for developing EPN include female sex, drug abuse, alcohol, neurogenic bladder and anatomical variations $[3,5,6]$. However the most significant risk factor is uncontrolled diabetes mellitus. The pathophysiology of this is thought to be caused by high level of glucose in the tissue as well as impaired blood supply to the kidney, both consequences of poorly controlled diabetes which facilitates anerobic metabolism required to develop EPN [1]. In this respect, this patient had a high number of risk factors being female, an insulin-dependent diabetic as well as her congenital renal disease.

EPN can be classified according to the location of gas within the renal system and extent of the disease using IVU or plain abdominal radiograph and diagnosis can be confirmed by ultrasound or CT scan. Huang and Tseng classify EPN into the following categories [2]: 1) gas in collecting system only; 2) gas in renal parenchyma without extension to extrarenal space; 3 ) extension of gas or abscess to perinephric space; extension of gas or abscess to pararenal space; 4) solitary kidney or bilateral disease.

Patients commonly present with dysuria, fever, rigors, flank pain, nausea and vomiting, all of which are symptoms consistent with pyelonephritis [4]. However more specifically to EPN, they may also present with leucocytosis, acute kidney injury, hematuria as well as heavy proteinuria [1]. This is a severe condition which can lead to death via sepsis; therefore it is important that there is appropriate initial management of these patients.

With regards to treatment, there are the options of medical management by giving antibiotics initially empirical antibiotics until urine culture reports are available, percutaneous drainage, nephrectomy whether it is emergency or delayed, or a combination of these methods can be initiated [6-8].

\section{Case Report}

A 43-year-old lady with background of insulin-dependent dia-

Articles (C) The authors | Journal compilation (C) World J Nephrol Urol and Elmer Press Inc ${ }^{\text {TM }}$ | www.wjnu.elmerpress.com

This is an open-access article distributed under the terms of the Creative Commons Attribution License, which permits unrestricted use, distribution, and reproduction in any medium, provided the original work is properly cited 


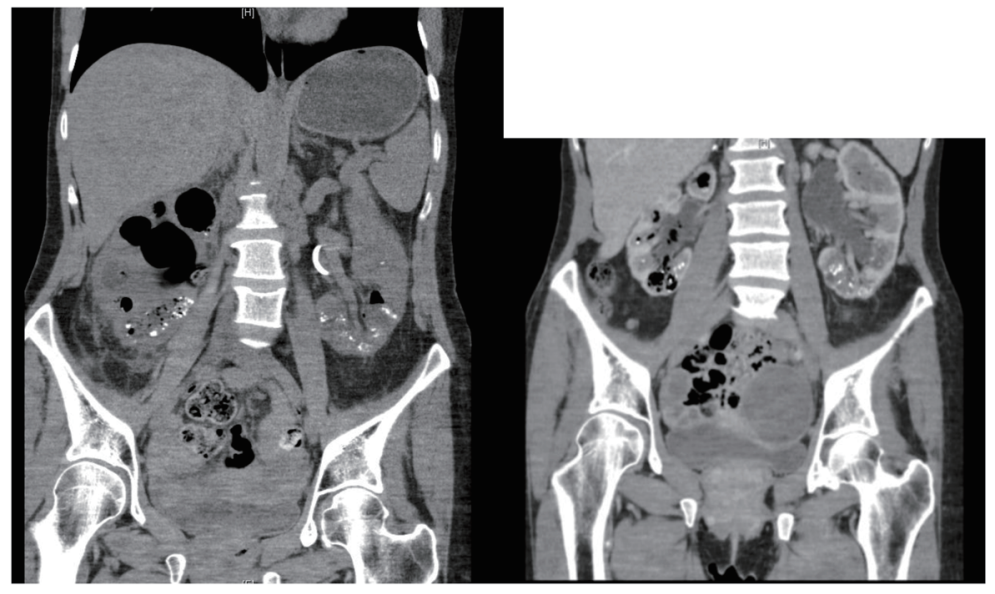

Figure 1. CT scans of EPN. Air evident in bladder and kidneys bilaterally before PCNL.

betes mellitus and medullary sponge kidney was referred with EPN, mild acute kidney injury and visible hematuria. She had had previous admissions for which she had been treated successfully with intravenous antibiotics.

On presentation, there was air in the bladder and urinary tract, namely the cortex and renal pelvis bilaterally, as well as stones in the collecting duct of the kidney and hydronephrosis. Initially she was completely asymptomatic and retrograde ureteropyelography demonstrated chronic EPN with capacious pelvicalyceal systems bilaterally. This was confirmed by CT scans showing extensive gas present in the collecting systems with inspissated debris particularly in the collecting system as well as calculi (Fig. 1). The ureters were tortuous, gas-filled and dilated with debris within the urine in the bladder as well as large quantities of gas in the bladder itself.

Air in the kidney or bladder is most commonly secondary to instrumentation, formation of fistulae or presence of gasproducing bacteria, all of which were not found in this patient. In light of the patient's acute kidney injury confirmed by her worsened renal function and sepsis, in addition to the matrix stones evident on scan causing multiple filling defects in the pelvic calyces and her worsening hydronephrosis with gas in the collecting system, nephrostomies were inserted to drain the kidneys bilaterally. Follow-up scans to review the need for ongoing drainage showed a heterogenous display in the lower pole and ureter bilaterally and uteroscopy was unsuccessful in relieving the obstruction. Microbiological analysis of the congealed material found in the kidney showed growth of Escheria coli and Brevibacterium species.

Since ureteroscopy was not successful, percutaneous nephrolithotomy (PCNL) although not conventionally used to treat EPN was attempted to clear the renal system, despite this usually was the method of treatment for large renal stones. The procedure was successful and follow-up interval CT KUB scans showed reduction in air compared to previous scans (Fig. 2).

There was no recurrence of matrix stones in the renal pelvices; she is clinically well but continues to have loin pain chronic in nature thought to be attributable to her bilateral medullary calcification in both kidneys secondary to her con-

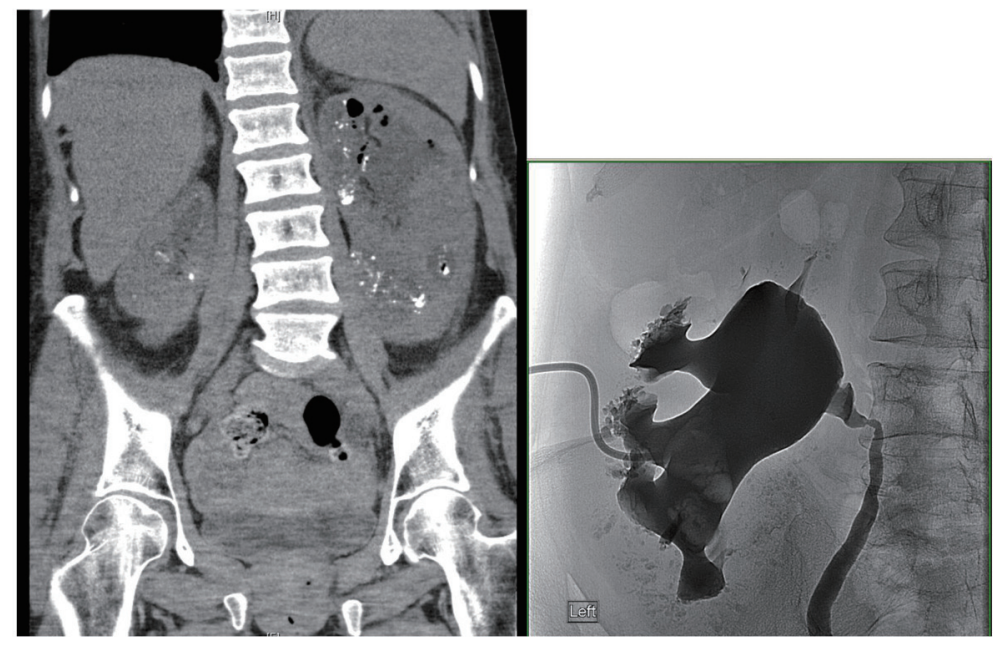

Figure 2. CT KUB after PCNL: reduction in air in kidneys. No matrix stones evident. 
genital medullary sponge kidney diagnosis.

\section{Discussion}

Several clinical findings are associated with poor outcome and higher mortality rate which includes thrombocytopenia, systolic pressure lower than $90 \mathrm{~mm} \mathrm{Hg}$, an altered level of consciousness, high level of serum creatinine and bilateral disease [5]. Initial prioritization should be to stabilize patients through providing oxygen and intravenous fluids for basic resuscitation in addition to correcting acid base imbalance and antibiotic coverage [1].

The difficulty lies in deciding the appropriate management of EPN for each patient since whereas previously there was a tendency to perform early nephrectomy, there is suggestion that percutaneous drainage may produce a better outcome. Research conducted by Yamaskai et al suggested that treatment could be determined by the extent of the disease. For example class I and II EPN had the best prognosis since the disease was limited and therefore could be resolved with medical management by antibiotics and percutaneous drainage or removal of urinary obstruction if present $[3,5]$. This is reiterated by Ubhee et al who suggested that percutaneous drainage should be applied if the gas was localized and there remained functioning renal tissue [1]. This is because emphasis should be resolution of EPN but also to preserve any functional renal tissue rather than the prophylactic previously preferred method of early nephrectomy. It is for this reason that percutaneous drainage is preferred since it helps preserve function of the affected kidney and the drainage tube can remain in situ until follow-up CT scan shows resolution $[1,8]$. However non-responders may still require nephrectomy.

Patients with class III and IV disease which was extensive because the disease would have extended beyond the renal capsule or was bilateral then management similar to that of class I and II could be applied or the patient should undergo early nephrectomy $[3,5]$. Other research suggested that early nephrectomy can also be used if there is no clinical progression or deterioration despite increased drain application since renal tissue would be unlikely to be salvage in these cases [4]. However Kapoor's study which although only involved a small cohort of 39 patients, showed that early nephrectomy led to a higher mortality rate than those that had percutaneous drainage with the latter group also having a higher rate of renal salvage [4]. Nevertheless studies show that those with extensive disease should be treated according to number of risk factors on clinical finding such as thrombocytopenia and altered consciousness with those with more than two risk factors undergoing nephrectomy whereas those with fewer having the same treatment as class I and II EPN with percutaneous drainage having the added benefit of drainage of pus and relief of gas pressure within the renal circulation [5].

\section{References}

1. Ubee SS, McGlynn L, Fordham M. Emphysematous pyelonephritis. BJU Int. 2011;107(9):1474-1478.

2. Huang JJ, Tseng CC. Emphysematous pyelonephritis: clinicoradiological classification, management, prognosis, and pathogenesis. Arch Intern Med. 2000;160(6):797805.

3. Wang JM, Lim HK, Pang KK. Emphysematous pyelonephritis. Scand J Urol Nephrol. 2007;41(3):223-229.

4. Kapoor R, Muruganandham K, Gulia AK, Singla M, Agrawal S, Mandhani A, Ansari MS, et al. Predictive factors for mortality and need for nephrectomy in patients with emphysematous pyelonephritis. BJU Int. 2010;105(7):986-989.

5. Yamasaki Y, Koga S, Minami Y, Sakai H. Emphysematous pyelonephritis with complete duplication of the left urinary tract. Can Urol Assoc J. 2011;5(6):E101-103.

6. Falagas ME, Alexiou VG, Giannopoulou KP, Siempos, II. Risk factors for mortality in patients with emphysematous pyelonephritis: a meta-analysis. J Urol. 2007;178(3 Pt 1):880-885; quiz 1129.

7. Derouiche A, Ouni A, Agrebi A, Slama A, Ben Slama MR, Chebil M. [Management of emphysematous pyelonephritis based on a series of 21 cases]. Prog Urol. 2008;18(2):102-107.

8. Somani BK, Nabi G, Thorpe P, Hussey J, Cook J, N'Dow $\mathrm{J}$. Is percutaneous drainage the new gold standard in the management of emphysematous pyelonephritis? Evidence from a systematic review. J Urol. 2008;179(5):18441849. 